\title{
Finite element method simulations of heat flow in fixed bed solar water splitting redox reactors
}

\author{
B. Bulfin*, B. E. Murphy, O. Lübben, S. A. Krasnikov, I. V. Shvets \\ School of Physics, Trinity College Dublin, Dublin 2, Ireland
}

\begin{abstract}
An improved design for radiation absorption and heat flow into materials with low thermal conductivity is demonstrated. The design was developed for application in fixed bed two step solar water splitting redox reactors. The fixed bed was assumed to be made from porous ceramic. The low thermal conductivity of the porous ceramic redox material is compensated for by changing the profile of the fixed bed. The profiling used was wedges cut into the material which allows concentrated solar radiation to be incident on a larger area of redox material than a flat monolith design. The design is demonstrated to efficiently transfer heat to the bulk and greatly reduce re-radiation. For a wedge $9 \mathrm{~cm}$ in depth and $1.6 \mathrm{~cm}$ wide at the opening, heated with $500 \mathrm{Wm}^{2} \mathrm{~s}^{-1}$ incident radiation for 300 seconds approximately double the amount of radiation is absorbed. The effects of thermal conductivity, emissivity and scaling of the design were investigated. The radiation absorption performance improved when scaled up. The improvement of the design over a flat plain increases for lower emissivity. The improvement provided by the wedge design was found to decrease for increasing thermal conductivity, and eventually for high conductivity values it reduced performance. Using this method a larger amount of material with low thermal conductivity can be heated with the same power input and reduced radiation losses. Finally a concentrated solar cavity reactor based on the design is proposed.
\end{abstract}

Keywords: Hydrogen production, Redox Reactors, Water Splitting, Efficiency, Heat Flow

\section{Introduction}

Splitting water to produce hydrogen using renewable energy sources is an attractive pathway for renewable fuels. A thermochemical water splitting cycle can be driven using concentrated solar power as the heat input [1, 2]. Thermochemical water splitting uses high temperature process heat to drive the endothermic water splitting reaction. Direct thermal water splitting is possible, but impractical as the process temperatures are very high ( $\approx 2500 \mathrm{~K}$ for partial decomposition). This decomposition leads to a high temperature mixture of gases which must be separated [3].

Two-step water splitting cycles proceed at lower temperatures, and hydrogen and oxygen are given off at different stages of the reaction, which removes the need for separating a mixture of gases[4]. Many two-step cycles have been proposed using metal oxides as the redox material [5]. In these cycles the process heat is used to reduce or partially reduce a metal oxide, releasing oxygen. The reduced oxide is then reacted with water to produce hydrogen.

The process can be described by the following chemical reactions

$$
\begin{aligned}
& M O_{x} \longrightarrow \mathrm{MO}_{x-\delta}+\frac{\delta}{2} \mathrm{O}_{2} \\
& M \mathrm{O}_{x-\delta}+\delta \mathrm{H}_{2} \mathrm{O} \longrightarrow \mathrm{MO}_{x}+\delta \mathrm{H}_{2}
\end{aligned}
$$

*Corresponding author. Telephone: +35318963139 email: bulfinb@tcd.ie
The first reaction is endothermic and requires high temperature process heat to proceed $\left(>1400{ }^{\circ} \mathrm{C}\right)$. The second reaction is slightly exothermic and proceeds at a lower temperatures $\left(\approx 900^{\circ} \mathrm{C}\right)$.

Thermodynamically zinc(II) oxide is one of the most promising materials [7]. However in practice the cycle has some complications. $\mathrm{ZnO}$ is volatile and sublimates when decomposing. This results in a hot mixture of zinc and oxygen gases which must be quenched to separate [8]. For this reason other cycles are sought with more simple separation processes.

The cycles we are concerned with here are those in which the redox material remains in the solid phase, but releases part of its oxygen. These cycles offer the simplest process as they can be used in a fixed bed reactor. Many promising materials have been identified including supported $\mathrm{NiFe}_{2} \mathrm{O}_{4}$ and $\mathrm{CeO}_{2}$ [9, 10, 11, 12]. Both of these compounds are partially reduced releasing some of their oxygen. Since they do not change phase, the oxygen is released through the surface, and the water splitting reaction takes place at the surface. In order to increase their yield the surface area must be maximised. To do this, the materials, either supported or unsupported, can be formed into porous monoliths [13, 14]. These monoliths form the fixed bed of the reactor.

The fixed bed of monolith is heated up to the reduction reaction temperature using concentrated solar power. The overall efficiency of the cycles, is largely determined by heat loss in the system, consequently the high operating temperature of such redox reactions and the length of time spent at high temperature 
can lead to large losses. For this reason, rapid reaction rates are preferable for the reduction step. The ratio of the amount of redox material to the power is also very important. If only a small amount of redox material can be heated by the concentrated solar radiation, then the chemical yield will be low compared to the amount of re-radiated heat. This presents a problem for fixed bed reactors made from porous ceramic monoliths.

The ceramic materials have low thermal conductivity, which is further reduced by the porosity [15]. This affects the heating rate of the fixed bed. The surface may be rapidly heated while the nearby interior, may take a long time to reach the reaction temperature. As the surface is heated rapidly, the fixed bed will re-radiate a lot of heat while the interior of the monolith is still heating up. This can result in large re-radiation energy loses, which greatly reduce the efficiency. The large temperature gradients can also degrade the materials due to thermal shock. It has been experimentally noted that if a large bulk porous monolith is used, the heating rate in the interior is very slow [16], while the surface will be rapidly heated and begin to re-radiate the received power. In order to compensate for this, thin layers of reactive material can be used. However, this means that only a small amount of redox material can be used and it will have a large re-radiating area.

Here we discuss a method of profiling the ceramic monoliths to reduce re-radiation and greatly increase the amount of oxide which can be cycled with a given power input. The oxide is also heated more uniformly which will reduce thermal shock degradation.

\section{Design}

The concept of the design considered is rather similar to that of a cavity. The geometry considered in this study is a block of redox material with wedges cut into the bulk material as seen in Fig. 1. Concentrated solar radiation then shines onto the wedges. This reduces the intensity received at the surface but to a certain extent traps the radiation. Most of the re-radiated heat is incident on the opposite surface. The tips of the wedges can potentially re-radiate a large amount of heat. However, the thermal conductivity of the porous ceramics is very low, and heat is not conducted from the interior of the ceramic to the tips of the wedges at a large rate, so the amount of heat which can be lost due to radiation from the tips is limited by the low transfer of heat from the interior.

The simulations of the design initially concentrate on an infinite flat plane with and without the wedges, in order to demonstrates the heat flow. A practical cavity receiver design is then discussed, which offers high radiation capture efficiency and very good heat flow into the fixed bed.

\section{Modeling}

All heat flow simulations were conducted using the Finite Element Method (FEM) [17]. The simulation solves the heat flow equation (Eqn. 3) over the constructed domains.

$$
C_{p} \rho \frac{\delta T}{\delta t}-k \Delta T=Q
$$

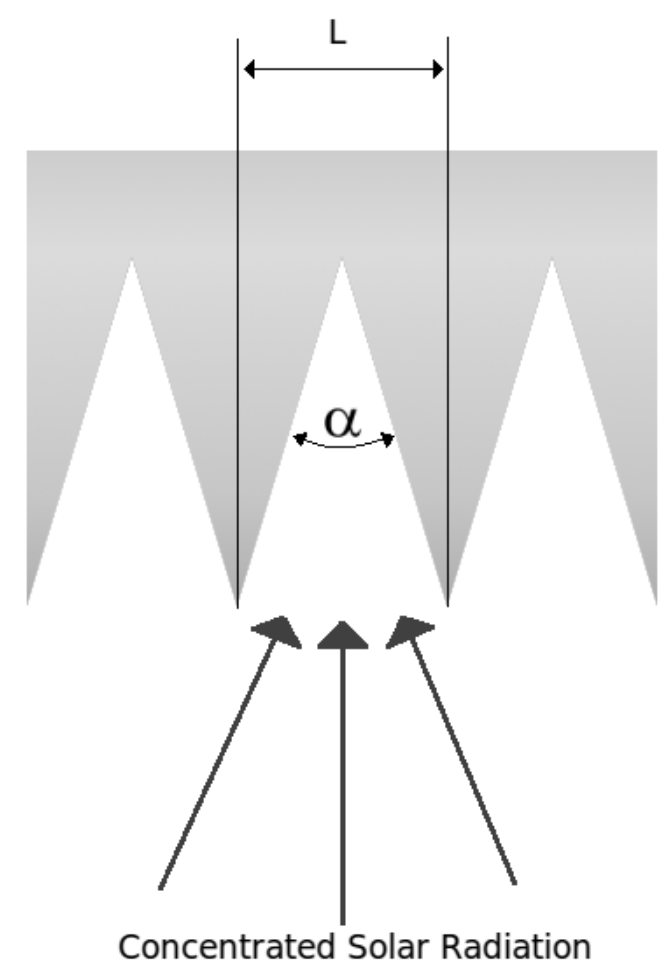

Figure 1: A cross section of the ceramic profile, where $\alpha$ is the acceptance angle for radiation in the plane of the page and $\mathrm{L}$ is the periodicity. The heat flow equations are symmetric about the parallel planes perpendicular to the page, indicated by the solid vertical lines.

Where $T$ is the temperature, $C_{p}$ is the specific heat, $\rho$ is the density, $k$ is the thermal conductivity and $Q$ is a heat source or sink term. In many reactor designs a method for reducing the oxygen partial pressure is used to stop the back reaction. This could involve placing the oxide in a purge gas or a vacuum. In this study we have considered the simple case of a vacuum. Now the only type of heat transfer at the boundaries is radiation from surface to surface or surface to ambient. The equations for heat flow at the boundaries are

$$
\begin{aligned}
& \hat{n} \bullet k \nabla T=q_{0}+\epsilon \sigma\left(T_{a}^{4}-T^{4}\right) \\
& \hat{n} \bullet k \nabla T=q_{0}+\left(G-\epsilon \sigma T^{4}\right)
\end{aligned}
$$

Equation 4 is the case where the surface is radiating to ambient and 5 is the case where there is surface to surface as well as surface to ambient radiation. Here $\hat{n}$ is the unit normal vector to the surface, $q_{0}$ is the surface heat source (concentrated solar radiation), $\epsilon$ is the emissivity of the surface, $T_{a}$ is the ambient temperature and $G$ is the surface irradiation (radiation received from other surfaces and ambient). The value of $G$ for a particular surface element is the sum of the radiation received by all of the other surface elements and the ambient. For example, for the $i t h$ surface element, $G_{i}$ would be of the form

$$
G_{i}=\sum_{i \neq j}^{j} F_{j i} \epsilon_{i} \epsilon_{j} A_{j} \sigma T_{j}^{4}+F_{a} A_{a} \epsilon_{i} T_{a}^{4}
$$

$F_{j i}$ and $F_{a}$ are the view factors. The view factor is the fraction of radiation leaving surface element $j$ that hits surface element 
$i$. They depend on the solid angle surface $j$ covers with respect to surface $i$, and the difference in angle between the line joining the surfaces and $j$ 's normal vector. $A_{j}$ and $A_{a}$ are the areas of the $j^{\text {th }}$ element and of the ambient. The sum for the surface to surface elements is over $j$ for $j \neq i$ to avoid self radiation. To calculate the view factors in the simulations the hemicube method was used [18].

The above equations form the basis for the model. The simulations were mainly conducted with a triangular mesh of finite elements in two dimensions (2D). The 2D simulations assume that the $2 \mathrm{D}$ profile extends to infinity, removing heat flow in that direction. The solution was linear between elements and computed using an iterative method.

\section{Results}

\subsection{Infinite Array of Wedges}

The initial simulations were carried out for an infinite flat plane with no additional measures to stop re-radiation. The acceptance angle in the plane of the page is the angle of the wedge $\alpha$, as seen in Fig. 1. In the plane perpendicular to the page radiation can be accepted from all directions.

The ceramic was modelled to have the heat capacity and density of $70 \%$ porous $\mathrm{CeO}_{2}$ [19]. The thermal conductivity of $\mathrm{CeO}_{2}$ and $\mathrm{NiFe}_{2} \mathrm{O}_{4}$ ceramics is very low, and when porosity is introduced it can more than half the thermal conductivity. We use an initial thermal conductivity of $2.5 \mathrm{Wm}^{-2} \mathrm{~K}^{-1}$ in the example below. (a)

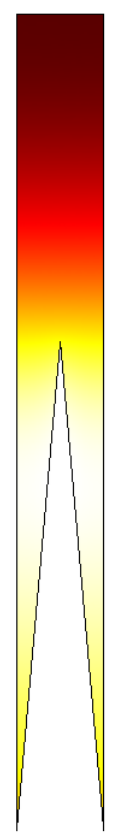

(b)

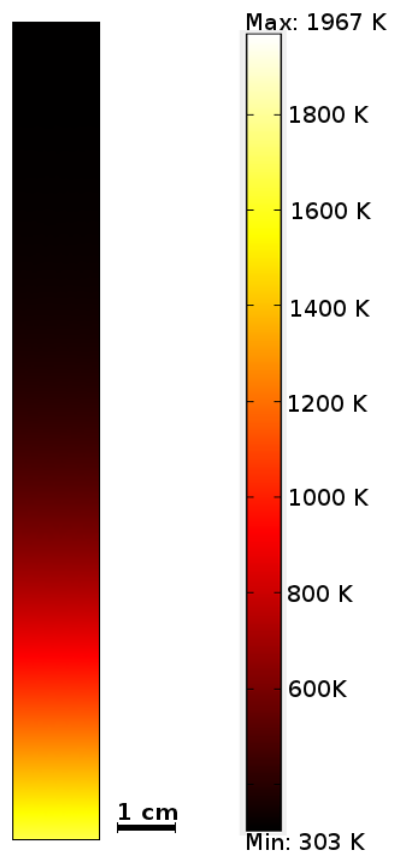

Figure 2: Temperature profiles for (a) the wedge design, and (b) a solid plane, after 300 seconds of heating from initial temperature of $300 \mathrm{~K}$

In Fig. 2 we see the plane with wedges analysed alongside a solid plane of the same material. The total thickness is $15 \mathrm{~cm}$, the wedge cut is $9 \mathrm{~cm}$ in depth and $1.6 \mathrm{~cm}$ wide at the opening. For simplicity, the material surfaces are assumed to radiate as black bodies $(\epsilon=1)$ to an ambient temperature of $300 \mathrm{~K}$. A power of $500 \mathrm{~kW} / \mathrm{m}^{2}$ is received by both surfaces, which is roughly 500 suns of concentration. The wedge receives much less heat per square metre of surface area however at a value of approximately $44 \mathrm{~kW} / \mathrm{m}^{2}$, due to the tilt of the receiving surface with respect to the radiation. As the wedge system is symmetric about the planes perpendicular to the page and bisecting the wedges at their tips, the solution will also be symmetric about these planes. For this reason they can be set to be thermally insulating forming a periodic boundary condition. The boundary at the back is also set to insulation as the heat lost here is negligible compared to the heat lost through the irradiated surfaces. This can be seen by the low temperature at the back surfaces even after 300 seconds of heating.

An immediate observation is that a much larger volume of ceramic is heated to high temperature in the wedge case. This is an excellent improvement as the chemical yield for the oxygen releasing step is directly proportional to the amount of material which is heated, which should increase the efficiency.

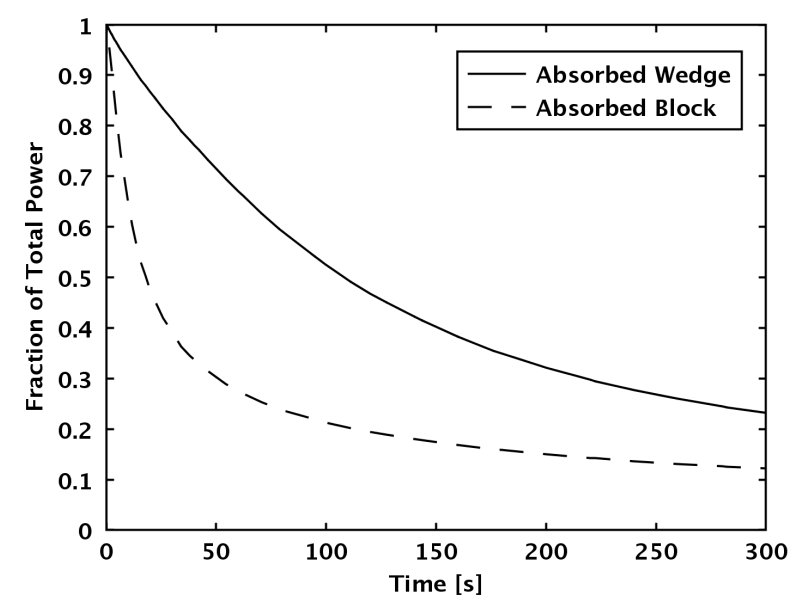

Figure 3: The fraction of the total power received at the surfaces which is not re-radiated by the wedge and block vs. time

In Fig. 3 the area below each curve is the fraction of the total energy supplied which is absorbed and the area above is the energy re-radiated. From this we can see that as time proceeds the amount of heat re-radiated by the wedge is far less than that re-radiated by the block. At 300 seconds, the temperature profiles are those shown in Fig. 2. The maximum temperature is reached in the interior of the wedges due to the re-radiation being trapped. The tips of the wedges are at relatively low temperature $(\approx 1370 \mathrm{~K})$ as compared to the surface of the solid plane $(\approx 1670 \mathrm{~K})$. This accounts for the lower amount of heat being re-radiated by the plane with the wedges cut.

\subsection{Effects of thermal conductivity, emissivity and scaling}

In order to investigate the ranges in which this geometry of redox material gives an improvement we have repeated the 
above analysis for different values of thermal conductivity, emissivity and scaling with an input power of $500 \mathrm{~kW} / \mathrm{m}^{2}$.

(a)
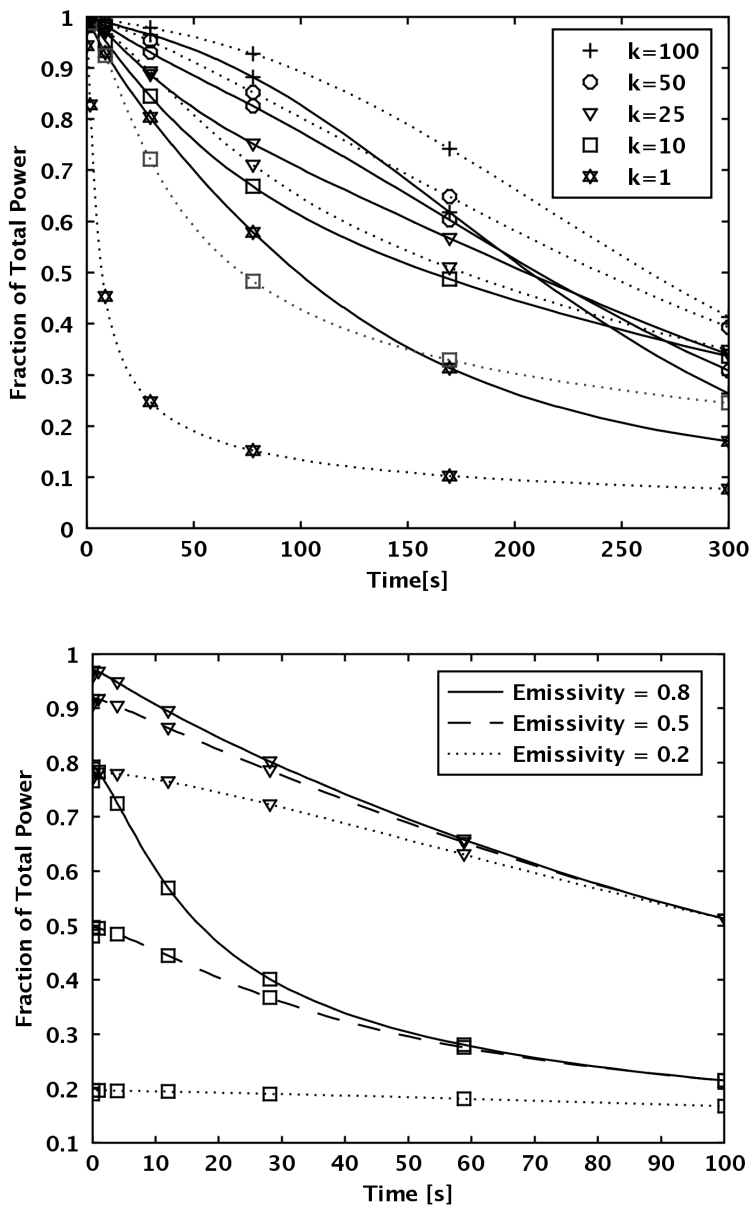

(b)

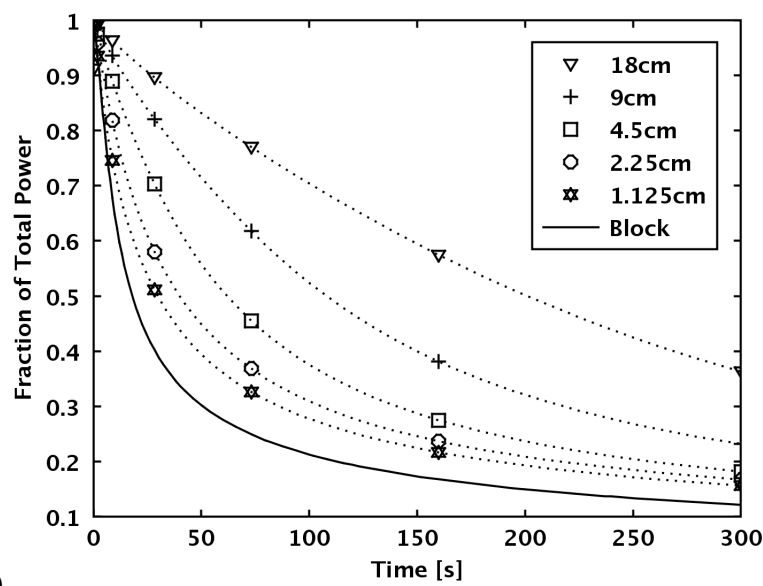

(c)

Figure 4: (a): The fraction of the total power received at the surfaces which is not re-radiated for different values of thermal conductivity. The solid lines are for the wedge and the doshed lines are the block. The dimensions of the wedge were those of Fig. 2. (b): The fraction of total power absorbed by the wedge and block for three different vaues of emissivity, where the triangular markers indicate the wedges and square markers indicate the block. (c): The fraction of the total power received at the surfaces which is not re-radiated for different depth wedges with the same acceptance angle.

From Fig. 4 (a) we can see that as the thermal conductiv- ity increases the amount of heat re-radiated decreases. For the block it decreases at a faster rate. For high values of $k>25 \mathrm{~W}$ $\mathrm{m}^{-2} \mathrm{~K}^{-1}$ the wedge set up begins to re-radiate more than the block. At high thermal conductivities the solid block is able to carry away the heat faster and the surface does not heat up as quickly. Therefore this method of improving radiation absorption is limited to the case of redox materials with low thermal conductivity.

At an intensity of $250 \mathrm{~kW} / \mathrm{m}^{2}$ the solid block outperforms the wedge when $\mathrm{k}>20 \mathrm{Wm}^{2} \mathrm{~K}^{-1}$. This lower threshold is due to the fact that the block needs to conduct less heat away from its surface. However, when the power is increased, the wedge design outperforms the block for a greater range of thermal conductivities.

Intuitively this makes sense, the tips of the wedges can reradiate a lot of heat, but if the thermal conductivity is low the power being supplied into the wedges cannot be conducted back to the tips. The wedge traps heat in the interior as most of the radiation is exchanged between the surfaces. The low thermal conductivity stops the heat from flowing to the highly radiating tips of the wedges and the lower the thermal conductivity the greater the improvement provided by the wedge system.

The affect of surface emissivity was also investigated. Fig. 4 (b) shows the fraction of power absorbed for three values of emissivity. The emissivity was constant over all frequencies. We can see that the block suffers badly with its initial power absorption scaling with the value of the emissivity. However in the wedge case the efficiency is not greatly decreased. Scattered light in the wedge case is likely to be incident on another portion of the wedge. Multiple scatterings result in much of the incident radiation being absorbed. This is a good sign as we would not expect the ceramic materials to act like ideal black bodies.

Finally the relationship between the scale of the wedges and the power absorption was examined. The depth of the wedge cut was varied between $1.125 \mathrm{~cm}$ and $18 \mathrm{~cm}$ with the acceptance angle kept constant at $\alpha=10.2^{\circ}$. Fig. 4 (c) shows that smaller scale wedges approach the performance of the block, as expected. However, for larger scale wedges, the radiation capture is further improved, and a larger amount of oxide is heated to the reaction temperature. This implies that implementation of this design in a large scale reactor could be feasible.

To get an idea of how this would affect the efficiency of a reduction reaction we can couple the heat flow equations to reaction kinetics and check the reaction efficiency. Using an Arrhenius rate equation made to match experimental decomposition of powdered $\mathrm{CeO}_{2}$ we can compare the reaction performance of the wedge to that of the block.

\subsection{Heat flow coupled to Arrhenius rate equation}

Using data extracted from an experiment in which $\mathrm{CeO}_{2}$ powder was decomposed in a vacuum, we can couple a rate law to our heat equations to evaluate potential performance of the wedge shaped reactor bed. Our decomposition reaction is

$$
\mathrm{CeO}_{2} \longrightarrow \mathrm{CeO}_{2-\delta}+\frac{\delta}{2} \mathrm{O}_{2}
$$


In the experiments a small amount of $\mathrm{CeO}_{2}$ nano-powder was rapidly heated in a vacuum using a focused Xenon lamp. The temperature and pressure were recorded and from this an Arrhenius rate equation was made to match the results. This is not an accurate representation of how the material would cycle if sintered into a porous monolith. The material was a nano powder with particle size less than $25 \mathrm{~nm}$ and thus had very high surface to volume ratio. The reactivity of a porous monolith would be lower. It is however useful to illustrate the potential for improvement in efficiency due to the wedge design.

The Arrhenius rate equations [20] used were of the form

$$
\begin{aligned}
& \frac{d\left[O_{o x}\right]}{d t}=k_{1}\left[O_{o x}\right]-k_{2}\left[O_{\text {gas }}\right] \\
& k_{x}=A_{x} e^{-} \frac{E_{x}}{R T}
\end{aligned}
$$

where $\left[O_{o x}\right]$ and $\left[O_{g a s}\right]$ are the concentrations of removable oxygen in the oxide and oxygen gas in the reaction chamber respectively. $A_{x}$ and $E_{x}$ are the frequency factors and activation energies which were varied to get a good match to the experiment. Only a small back reaction term was included as in this case we assume that the vacuum is being maintained.

The reaction consumes heat so we now have a heat sink term in the interior. The heat sink takes the form

$$
Q=-\frac{d\left[O_{o x}\right]}{d t} * \Delta H
$$

where $\Delta H$ is the change in enthalpy of the reaction. The value of $\Delta H$ varies from $963 \mathrm{~kJ} / \mathrm{mol}_{O_{2}}$ to $770 \mathrm{~kJ} / \mathrm{mol}_{O_{2}}$ as $\delta$ is varied from 0 to 0.2 [21]. For simplicity, the weighted average of around $800 \mathrm{~kJ} / \mathrm{mol}_{\mathrm{O}_{2}}$ was used. The maximum $\delta$ in these simulations was set to 0.2 , in agreement with our results. This value seems a little high when compared to the literature [21], and may have be attributed to the very high surface area of the nano-particles.

The initial temperature in the simulation was changed from $300 \mathrm{~K}$ to $1000 \mathrm{~K}$, to simulate a cycle situation, where the second step takes place at lower but not ambient temperature. If continuously cycled the material temperature should not drop below $1000 \mathrm{~K}$ due to self heating in the water splitting reaction.

In Fig. 5 the red curves are for the wedge and the blue are for the solid block. The solid lines are the fraction of the incoming power which is not re-radiated and dashed lines are the fraction of the total power consumed by the reaction. The energy consumed by the reaction in the case of the wedge is far higher than that of the block. This is due to the larger quantity of the reactive ceramic heated. This reduces the amount of re-radiated heat as it consumes heat, reducing the temperature of the reactive ceramic, which can be seen by the bulge that appears in the absorption curve, compared to Fig. 3. This is an excellent improvement over the case of the block where the large majority of the incoming power is re-radiated and only a small amount of the ceramic is reduced.

It is also notable that when the thermal conductivity is reduced to

$0.5 \mathrm{Wm}^{-2} \mathrm{~K}^{-1}$, the reaction efficiency of the block is approximately halved while the performance of the wedge is almost

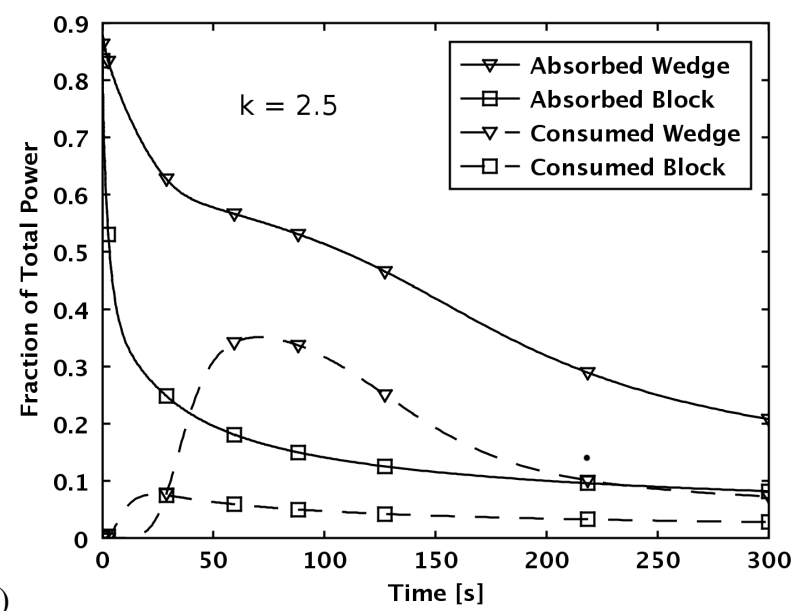

(a)

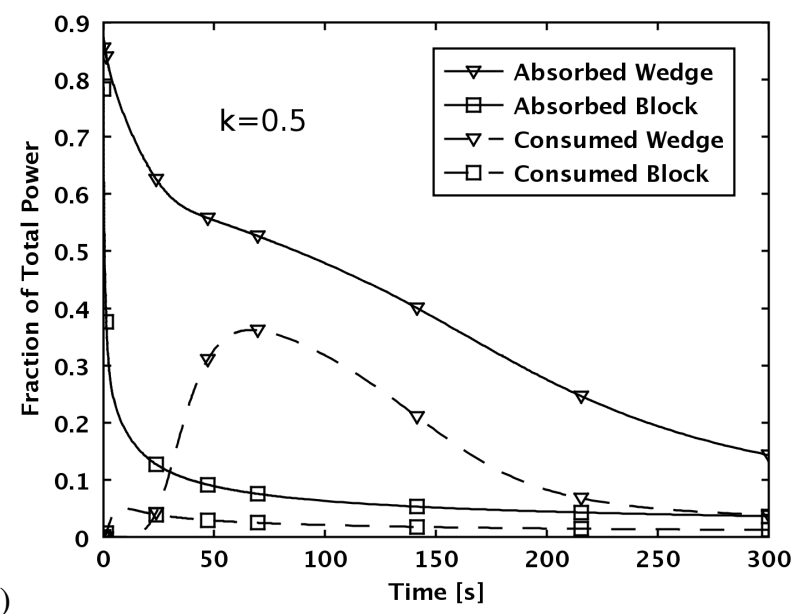

Figure 5: Total energy absorbed and consumed by the reaction for the wedge and block for two values of thermal conductivity, where the lines marked with triangles are for the wedge and those marked with squares are for the block. The solid lines are the absorption and the dashed lines are the consumed power.

unaffected. This result is promising for the porous ceramics as their thermal conductivity could be very low.

\section{Efficient Cavity Receiver Design}

In order to design an efficient cavity receiver we must ensure that radiation received into the cavity is inside the acceptance angle of the wedges. This is not difficult as we can simply set the minimum acceptance angle to be the angle made between the material and the cavity opening. This is shown in Fig. 6 for a cavity using a compound parabolic concentrator, where $\alpha$ is the minimum acceptance angle for a wedge.

As an example we have simulated a two dimensional cavity receiver with and without wedges. The same reaction equations and material properties as section 4.3 were used. The thermal conductivity was again set to $2.5 \mathrm{Wm}^{-2} \mathrm{~K}^{-1}$. The total outside width of the cavity is $80 \mathrm{~cm}$, the height is $45 \mathrm{~cm}$, and the input aperture is $8 \mathrm{~cm}$. The input power through the aperture was $1500 \mathrm{Wm}^{-2} \mathrm{~s}^{-1}$ which is equivalent to a concentration of 1500 suns. The interior was assumed to be a vacuum and the external walls are set to thermal insulataion for simplicity. Fig. 7 shows 


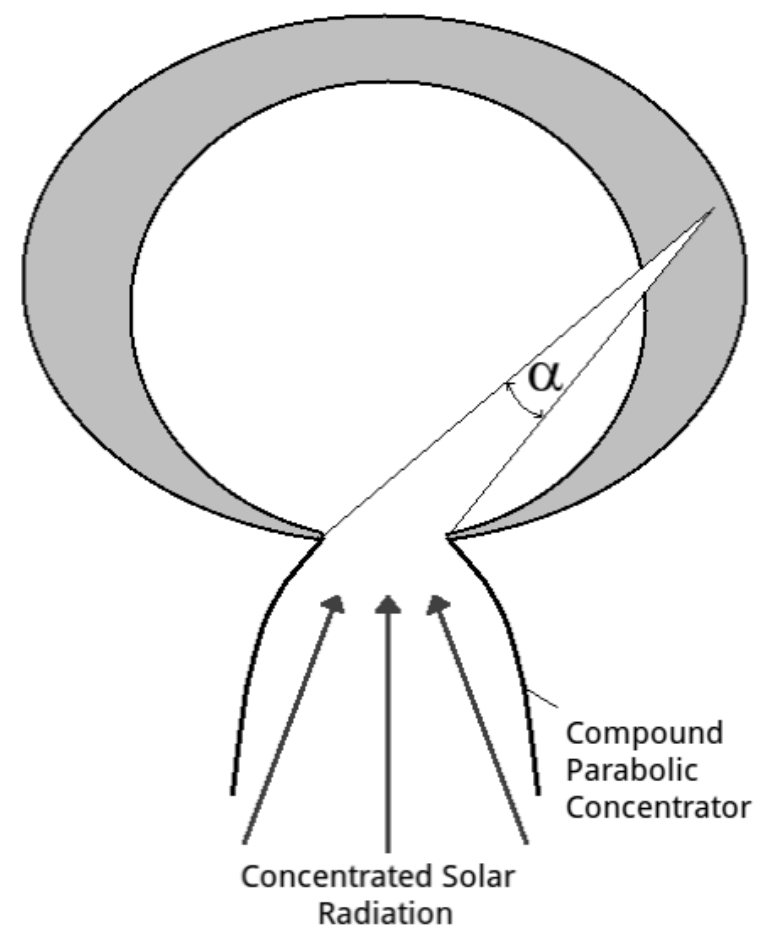

Figure 6: A cross section of a cavity showing one wedge cut.

the temperature profiles and the power which is absorbed and sonsumed in both cases.

An immediate observation is that the wedge case is far more uniformly heated. This is good as thermal shock will be reduced which will improve material lifetimes. The wedge case uses roughly double the fraction of the power used as compared to the flat interior cavity. It is however yet to be determined weather this wedge design can have such an improvement for a three dimensional cavity design. The authors leave this for future work.

\section{Discussion}

The design could be adapted to improve the efficiency of a number of fixed bed reactor designs. In order to fully determine its potential the optical properties of the porous monolith material would need to be determined. If the material acted reasonably close to a black body (like $\mathrm{Fe}_{3} \mathrm{O}_{4}$ ) the analysis would be a reasonably good measure of the materials performance.

These simulations illustrate the importance of optimising heat flow to improve reactor efficiencies. In order to improve the efficiency, one needs to increase the yield, reduce radiation loses and exchange the excess heat from the reduction step of the cycle. We see from this that the low thermal conductivity of the porous ceramics is problematic but can be designed around.

In our calculations we assumed that the materials were in a vacuum. In the case of a purge gas being used, and provided that $P>>V_{t} \int_{T_{i}}^{T_{f}} C_{v}(T) d T$, then the result should not greatly differ. Here $P$ is the power supplied to the reactor, $V_{t}$ is the volumetric flow rate of the purge gas, $T_{i}$ its initial temperature,
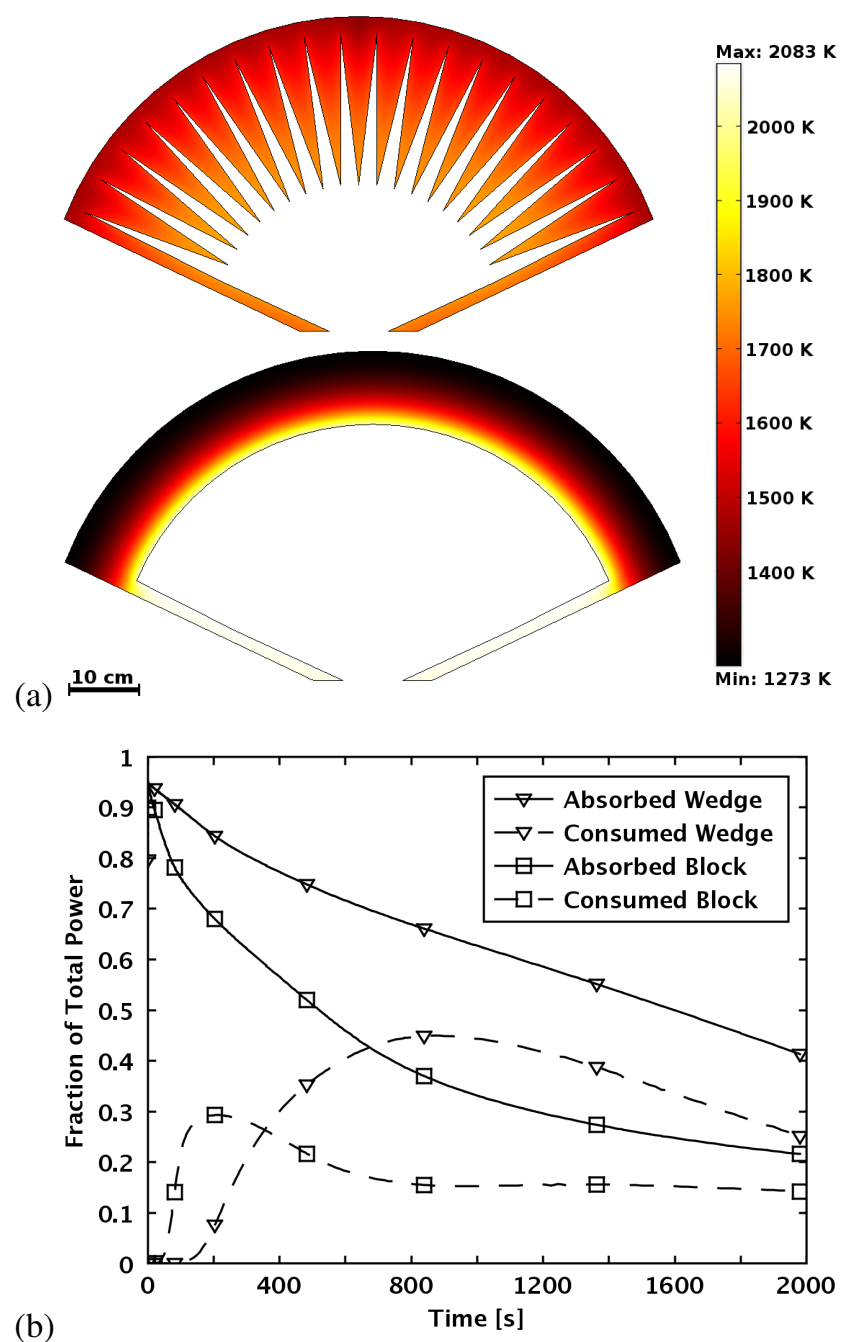

Figure 7: (a): Temperature profile of a two dimensional cavity with and without wedges after 15 minutes of heating. (b): Fraction of total power absorbed and consumed over 30 minutes for the two cavities where again the lines marked with triangles denote the cavity with wedges. Solid lines are for absorption and dashed lines for consumption.

$T_{f}$ its final temperature and $C_{v}$ the volumetric specific heat capacity. If the purge gas was flowing from the hot area to the cold area of the monolith it would also improve the heat flow through the ceramic.

\section{Conclusions}

Our FEM simulations show that cutting wedges into low thermal conductivity materials allows larger quantities of the material to be heated with concentrated solar power and reduces reradiation losses. For the example used the absorption efficiency was approximately doubled over 300 seconds of heating. This improvement is restricted to cases of low thermal conductivity. The range of values of thermal conductivity for which it offers an improvement increases with increasing incident power. For an input power of $500 \mathrm{Wm}^{-2} \mathrm{~s}^{-1}$ the wedges improved absorption when the thermal conductivity was less than $25 \mathrm{~W} \mathrm{~m}^{-2}$ $\mathrm{K}^{-1}$. For low values of surface emissivity the wedge further 
outperforms the block due to multiple reflections of light in the wedge. The design can be scaled up to further improve the radiation absorption performance. When used in a vacuum environment to reduce reactive ceramics the reaction yield and efficiency is significantly improved due to more material being heated and less re-radiation.

\section{Acknowledgments}

This work has received funding from SFI-06/IN1/I91, and the International Graduate Research Programme in Micro- \& Nano Engineering, an IRCSET graduate research education programme. It was conducted in association with the Cleaner Energy Lab Trinity College Dublin. This article has been published in the International Journal of Hydrogen Energy. It is also available at elsevier http://www.sciencedirect.com/science/article/pii/S0360319912008518

[1] T. Kodama. High-temperature solar chemistry for converting solar heat to chemical fuels. Progr Energy Combust Sci, 2003;29:567-97.

[2] Aldo Steinfeld. Solar thermochemical production of hydrogena review. Sol Energy, 2005;78:603-15.

[3] S.Z. Baykara. Experimental solar water thermolysis. Int $\mathbf{J}$ Hydrogen Energy, 2004;29:1459-69.

[4] Tatsuya Kodama and Nobuyuki Gokon. Thermochemical cycles for hightemperature solar hydrogen production. Chem Rev, 2007;107:4048-77.

[5] Stphane Abanades, Patrice Charvin, Gilles Flamant, and Pierre Neveu. Screening of water-splitting thermochemical cycles potentially attractive for hydrogen production by concentrated solar energy. Energy, 2006;31:2805-22.

[6] Christopher Perkins and Alan W. Weimer. Likely near-term solar-thermal water splitting technologies. Int J Hydrogen Energy, 2004;29:1587-99.

[7] Irina Vishnevetsky, Alexander Berman, and Michael Epstein. Features of solar thermochemical redox cycles for hydrogen production from water as a function of reactants main characteristics. Int $\mathbf{J}$ Hydrogen Energy, 2001;36:2817-30.

[8] A. Steinfeld. Solar hydrogen production via a two-step water-splitting thermochemical cycle based on $\mathrm{Zn} / \mathrm{ZnO}$ redox reactions. Int J Hydrogen Energy, 2002;27:611-19.

[9] Fernando Fresno, Tomoaki Yoshida, Nobuyuki Gokon, Roco FernndezSaavedra, and Tatsuya Kodama. Comparative study of the activity of nickel ferrites for solar hydrogen production by two-step thermochemical cycles. Int J Hydrogen Energy, 2010;35:8503-10.

[10] Hiroshi Kaneko, Takao Miura, Akinori Fuse, Hideyuki Ishihara, Shunpei Taku, Hiroaki Fukuzumi, et al. Rotary-type solar reactor for solar hydrogen production with two-step water splitting process. Energy Fuels, 2007;21:2287-93

[11] Alex Le Gal and Stphane Abanades. Catalytic investigation of ceriazirconia solid solutions for solar hydrogen production. Int $\mathrm{J}$ Hydrogen Energy, 2011;36:4739-48.

[12] H. Kaneko, T. Kodama, N. Gokon, Y. Tamaura, K. Lovegrove, and A. Luzzi. Decomposition of $\mathrm{Zn}$-ferrite for $\mathrm{O}_{2}$ generation by concentrated solar radiation. Sol Energy, 2004;76:317-22.

[13] Nobuyuki Gokon, Tatsuya Kodama, Nobuki Imaizumi, Jun Umeda, and Taebeom Seo. Ferrite/zirconia-coated foam device prepared by spin coating for solar demonstration of thermochemical water-splitting. Int $\mathrm{J}$ of Hydrogen Energy, 2001;36:2014-28.

[14] James Miller, Mark Allendorf, Richard Diver, Lindsey Evans, Nathan Siegel, and John Stuecker. Metal oxide composites and structures for ultra-high temperature solar thermochemical cycles. J Mat Sci, 2008;43:4714-28.

[15] T.H. Bauer. A general analytical approach toward the thermal conductivity of porous media. Int J Heat Mass Transfer, 1993;36:4181-91.

[16] William C. Chueh, Christoph Falter, Mandy Abbott, Danien Scipio, Philipp Furler, Sossina M. Haile, et al. High-flux solar-driven thermochemical dissociation of $\mathrm{CO}_{2}$ and $\mathrm{H}_{2} \mathrm{O}$ using nonstoichiometric ceria. Science, 2010;330:1797-801.
[17] Roland Fortunier Jean-Michel Bergheau. Finite Element Simulation of Heat Transfer. Wiley; 2004.

[18] Micheal W. Glass. Chaparral: A library for solving large enclosure radiation heat transfer problems. Technical Report SAND95-2049 UC-700, Sandia National Laboratories, East Lansing, Michigan.

[19] Comsol Multiphysics. Heat transfer user's guide, version 4.2. 2011.

[20] Moungi Gabriel Bawendi Robert J. Silbey, Robert A. Alberty. Physical chemistry, 4th Ed. Wiley; 2005.

[21] R.J. Panlener, R.N. Blumenthal, and J.E. Garnier. A thermodynamic study of nonstoichiometric cerium dioxide. J Phys Chem Solids, 1975;36:121322. 